\title{
PRÁCTICA FILOSÓFICA CON ACCIONES CORPORALES
}

\section{PHILOSOPHICAL PRACTICE WITH CORPORAL ACTIONS}

\author{
DAVID SUMIACHER D'ANGELO ${ }^{1}$ \\ Universidad Nacional Autónoma de México - Centro Educativo \\ para la Creación Autónoma en Prácticas Filosóficas (CECAPFI) \\ México \\ davosum@gmail.com
}

RECIBIDO: 22 DE ABRIL DE 2020

ACEPTADO: 2 DE NOVIEMBRE DE 2020

Resumen: En este artículo brindaré algunos elementos y perspectivas respecto a cómo es posible la realización de prácticas filosóficas a través de acciones corporales. Para entender esto y poder llevarlo a cabo primeramente debemos entender algunos presupuestos y principios generales en torno a una comprensión amplia de la filosofía y la práctica filosófica. Luego de asentar estas bases, me concentraré en tres grandes formas de realizar acciones corporales como parte del trabajo que se realiza en la práctica filosófica. La primera tiene que ver con la captación sensorial del otro. De esta manera enriquecemos enormemente el caudal de elementos que utilizamos para el trabajo con los demás y el desarrollo de hipótesis de trabajo. Un segundo tipo de acciones corporales que podemos incorporar en la práctica filosófica, tiene que ver con la realización de acciones corporales por parte del filósofo práctico. En este caso utilizamos nuestra acción, gesticulación y expresiones como modo de propiciar, profundizar e intensificar procesos filosóficos que se desarrollen a través del diálogo o de cualquier forma. Por último, una tercer forma de inclusión de la acción corporal en el devenir filosófico intersubjetivo, tiene que ver con solicitar la realización de acciones corporales específicas a los otros o de realizarlas en conjunto con ellos. La variedad de posibilidades y de rutas posibles en este caso es inmensa, pero he aquí un potencial indiscutible para el trabajo filosófico con otros, que se construye en principio a partir de la captación de la circunstancia y del proceso filosófico que se venga desarrollando. En cada caso estaré considerando una

${ }^{1} \mathrm{El}$ autor no dispone de registro ORCID 
visión amplia de la práctica filosófica que incluye la labor de la consultoria filosofica, la filosofía con/para para niños, los talleres filosóficos y la filosofía aplicada en organizaciones.

Palabras clave: práctica filosófica, acciones, acciones corporales, consultoría Filosófica, Filosofia para Niños, filosofia con niños, talleres filosóficos, filosofía en organizaciones.

Abstract: In this article I will provide some elements and perspectives regarding how it's possible to carry out philosophical practices through corporal actions. To understand this and to be able to do it out first, we must understand some general assumptions and principles around a broad understanding of philosophy and philosophical practice. After laying these foundations, I will focus on three great ways of performing corporal actions as part of the work that is done in philosophical practice. The first has to do with the sensory uptake of the other. In this way we enormously enrich the flow of elements that we use for working with others and the development of working hypotheses. A second type of corporal actions that we can incorporate into philosophical practice has to do with the performance of bodily actions by the practical philosopher. In this case, we use our action, gestures and expressions as a way of promoting, deepening and intensifying philosophical processes that are developed through dialogue or in any way. Lastly, a third way of including corporal actions in intersubjective philosophical interactions has to do with requesting the performance of specific corporal actions from others or performing them in conjunction with them. The variety of possibilities and achievable routes in this case is immense, but here we have an indisputable potential for philosophical work with others, which is built in principle from the grasp of circumstance and the philosophical process that is developing. In each case I will be considering a broad vision of philosophical practice that includes the work of philosophical counseling, philosophy for/with children, philosophical workshops and applied philosophy in organizations.

Key words: philosophical practice, actions, corporal actions, philosophical counseling, Philosophy for Children, philosophy with children, philosophical workshops, philosophy in organizations. 


\section{Introducción ${ }^{2}$}

Para comenzar con estas ideas, tengo que referir a algunos elementos epistemológicos de manera algo general. Muchas veces se piensa que la filosofía se define por el pensar, hacer uso del lenguaje, hacer buenas preguntas o desarrollar nuestro "pensamiento crítico". Ciertamente todas estas cosas pueden sin duda ser parte de la filosofía, tocan diversos ámbitos de lo que esta es o puede hacer pero no abarcan, ninguna de ellas, lo que la filosofía es. Me atrevo a hacer afirmaciones tan fuertes desde el inicio porque tengo la certeza de que nadie (o casi nadie) desea un quehacer filosófico que se refugie únicamente en el marco del "pensar". A todos nos interesan las ideas cuando se relacionan con el mundo en que vivimos. Aunque las preguntas puedan ser sumamente importantes y gran parte de la filosofía y la práctica filosófica se nutra de ellas, ellas solo generan, en un principio, pensamiento. Una pregunta puede sin duda moverme a actuar, pero solo si primero la decodifico, la analizo y luego tomo una decisión. Únicamente a partir de entonces es que una pregunta se relaciona con una acción. La acción es una cosa distinta a la pregunta, posterior pero no implicada en la primera. Quiero decir, la pregunta es de sumo valor, pero sin tanta dificultad podríamos imaginarnos circunstancias en donde lo importante -es actuar-, no preguntarnos

${ }^{2}$ La forma de citado de este texto corresponde a un posicionamiento respecto a la lectura y a la relación con las fuentes. Todas las fuentes referidas en el cuerpo del texto aparecen con el nombre de la obra y del autor originales, pero con el lugar y fecha de escritura de la primera edición. Esto tiene que ver con el concepto destacado por el filósofo Enrique Dussel de locus enuntiationi (lugar de enunciación) de la obra en cuestión, lo que brinda al lector una referencia acerca de quién, dónde y cuándo afirmó aquello que aquí se menciona. Esto se realiza con la función de comprender el origen de las ideas desde un marco concreto, real e histórico y no solo como pensamientos desprovistos de localización. Posteriormente en el apartado final de bibliografía se referencian las ediciones consultadas con la fecha, lugar y editorial o referencia respectiva. 
y justamente esa acción o reacción diferente a la pregunta podría ser la cosa más trascendente y filosófica que pudiera realizarse. En palabras del gran pedagogo Paulo Freire: "debo utitlizar todas las posibilidades a mi alcance, no solo para hablar de mi utopía, sino para participar en prácticas coherentes con ella" 3 .

Finalmente nadie creería a un "teórico" de la justicia que no es justo en sus acciones a un teórico de la amistad que no puede tener ningún amigo. ¿Cuál de estos personajes es el que nos interesa? Aunque la importancia de la teoría es innegable, evidentemente su único valor está dado gracias a la posibilidad de que nosotros tenemos de vincularnos con "las cosas a las que la teoría refiere". Hablar sobre la justicia o la amistad tiene sentido gracias al amplio grupo de acciones justas o amistosas que existen o podrían existir. Con el lenguaje y el pensamiento crítico es una cuestión bastante similar. De nada me sirve su existencia sin que los sujetos accionen y transformen su modo de vida. ¿Para que hablar o pensar sobre "el encierro", "la coherencia", "la armonía", "la violencia", "la hospitalidad", "el acontecimiento", etc. si estos no están o pudieran estar en el mundo? ${ }^{4}$. Si así fuera, la filosofía simplemente podría hablar sobre "cualquier tema" y no tendría entonces ningún interés social.

Pero la filosofía nace, y no solo eso, se realiza en conjunto en el mundo de nuestras acciones corporales así como de toda la procesualidad viva que somos. Atraviesa nuestra vida como coherencias procedimentales, como corrientes de interconexión,

\footnotetext{
${ }^{3}$ Freire, Paulo: Pedagogía de la indignación. Cartas pedagógicas en un mundo revuelto, Siglo XXI, Buenos Aires, 2012, p. 39.

${ }^{4}$ A esto mismo refería el lógico mexicano Raymundo Morado cuando afirmaba que la lógica es una ciencia pero a la vez un arte, y es un arte porque debemos aplicarla también en la vida cotidiana. (Esto en relación al video publicado recientemente por la Academia Mexicana de Lógica: https://www.youtube.com/watch?v=3wrMuY-9WMc (consultado el 25 de marzo de 2020).
} 
como cursos de existencia que se relacionan con el sentido que podemos dar y vivir en nuestro devenir humano. La filosofía pasa, sucede -dentro del juego- ${ }^{5}$, no solo en nuestra mente. Así puede decirse que la filosofía existe o está presente en distintos ámbitos de nuestra vida, y lo más lógico es que lo esté más en unos que en otros. A veces nos encontramos con niveles filosóficos casi cercanos a cero, otras veces la filosofía irrumpe con gran vigor en la vida nuestra y en el entorno. Pero incluso aunque parezca que algo que alguien hace no tiene nada en absoluto de filosófico, basta ver con un poco más de cuidado para notar que todo los actos que realizamos poseen, aunque sea, un grado mínimo de filosofía, al menos en forma "germinal" . Esto es importante para la consultoría filosófica o la práctica filosófica en general, ya que nosotros trabajamos con todo tipo de personas y situaciones. También es cierto que un acto posea siempre una dimensión filosófica en forma germinal no significa que la persona, grupo o sociedad en cuestión desee o finalmente logre manifestar esa posiblidad filosófica. Eso depende de muchos factores y uno de los principales es la voluntad y determinación de nuestros invitados a la práctica filosófica. La filosofía no es a priori de facto, es un a priori siempre como posibilidad.

El punto es que este gran centramiento o interés que la filosofía ha tenido por los procesos de pensamiento y o del lenguaje, ha

\footnotetext{
${ }^{5}$ Husserl, en este mismo sentido, proponía una re-consideracion de la doxa para la episteme (Husserl, Edmund: La crisis de las ciencias europeas y la fenomenología trascendental, Crítica, Barcelona, 1991, p. 164), Merleau-Ponty decía que la filosofía no era el reflejo de una verdad previa sino la "realización de una verdad" (Merleau-Ponty, Maurice: Fenomenología de la percepción, Planeta, España, 1994, p. 20).

${ }^{6}$ Evidentemente no puedo ahondar aquí en estas ideas, si se quisiera profundizar en ello invito a consultar: Sumiacher, David: "La filosofía como una expansión de la vida y los procesos", en Sumiacher, David: Prácticas filosóficas comparadas, Novedades Educativas/Editorial CECAPFI, Buenos Aires, 2020, pp.121-132.
} 
arrastrado también a la práctica filosófica a una tendencia análoga. Las acciones corporales han sido casi olvidadas en la práctica filosófica y a veces incluso en la vida misma de los filósofos. Pero las acciones corporales tienen un gran potencial, un potencial insustituible. Desde esta perspectiva, una acción es una vinculación con la exterioridad ${ }^{7}$. Estas, a su vez, pueden ser de carácter activo o receptivo. Activo cuando infrinjen, transforman, afectan el entorno y receptivas cuando, utilizando los sentidos, perciben el mundo. Al mismo tiempo, además de las acciones corporales existen las acciones discursivas, sobre las que bastante se ha analizado, por ejemplo un representante predilecto en el tratamiento de esto es John Austin ${ }^{8}$. Este tipo de acción se compone básicamente del decir y el ecuchar según sea activa o receptiva. Pero más allá de las acciones corporales y las acciones discursivas no hay ningún otro tipo de acción existente dentro de las posibilidades del humano tal como hoy lo conocemos. Como de las acciones discursivas ya se ha hablado mucho, se conoce bastante y también son muy utilizadas dentro del campo de la filosofía y la práctica filosófica, dediquémonos a pensar qué sucede y cómo podemos utilizar el gran potencial de las acciones corporales para este quehacer. Adentrémonos a pensar cómo la acción corporal puede beneficiar o enriquecer el trabajo de la consultoría filosófica, los talleres filosóficos, la filosofía con/para niños o la filosofía en organizaciones.

\footnotetext{
${ }^{7}$ Este tipo de ideas pueden consultarse en: Sumiacher, David: "Acts, processes, thought and action in philosophical practice", en Amir, Lydia: New Frontiers of Philosophical Practice: Expanding Boundaries, Cambridge, UK, 2017, pp.142160.

${ }^{8}$ Uno de sus clásicos textos sobre el tema es: Austin, John: Cómo hacer cosas con palabras, Ediciones Paidos, Barcelona, 1982. También en general esto puede investigarse relacionándose con toda la gama de teorías contemporáneas de la lógica informal, teoría de la argumentación o pensamiento crítico.
} 
El primer nivel que aquí voy a presentar consiste en observar o percibir a nuestro(s) interlocutor(es) y los actos que realiza(n) para hacer uso de estas percepciones dentro del proceso de la práctica filosófica. La sensibilidad a las expresiones de la subjetividad del otro es en general una de las capacidades más importantes de aquel que se dedica a hacer este tipo de labor. Dentro de ella está la captación de gestos o movimientos, es decir, acciones corporales efectuadas por nuestro consultante, grupo o personas de la organización con la que estemos trabajando. Entrenando esta sensibilidad, y logrando captar estas acciones como parte de la procesualidad significativa en el otro, puedo luego hacer diferentes cosas. No siempre una vez que observe una actitud o acción interesante, será el momento de enunciar algo respecto, en ocasiones solamente se trata de retenerlo para externarlo después, conectado con otros elementos que puedan dar más fuerza argumentativa a la circunstancia o al proceso filosófico que se esté trabajando. Pero si tienen significatividad, tarde o temprano, esos elementos observados tienen que ser utilizados por el consultor o filósofo práctico, más aún si estos se repiten y se vinculan a algo similar a lo que Ran Lahav llama "patrones".

Como sea, este es el primer nivel de acercamiento con las acciones corporales en la práctica filosófica, es el nivel en donde el filósofo práctico observa las acciones corporales del otro y las utiliza, las piensa y conecta con el proceso de construcción de hipótesis; o las devuelve -cuestionando lo que el otro hace-: “¿Por qué cuando

\footnotetext{
${ }^{9}$ Véase el Cap 4 de Lahav, Ran: Saliendo de la caverna de Platón, Loyev Books, Vermont, 2016. Es complejo en este espacio realizar una comparación entre las ideas de este interesante pensador israelí y esta propuesta. A resumidas cuentas, no son -únicamente- los patrones de Lahav el parámetro para tener en cuenta o no una acción corporal respecto a un proceso filosófico. Existe una serie de procesos filosóficos de carácter creativo que no tienen que ver directamente con los patrones, porque no reflejan una dimensión de límite o restricción para el sujeto y sin embargo reportan una gran significatividad.
} 
estaba diciendo lo que dije cambió la forma de su mirada?”, “¿Por qué elevó/le tembló la voz?”, “¿Por qué sus brazos comenzaron a moverse más ampliamente cuando me expresó ese punto?". Mi perspectiva no adhiere a la idea del "lenguaje no verbal". Para mí el lenguaje siempre es un proceso compuesto, es decir, una actividad combinada en donde hay una cosa que refiere a otra cosa. Todo lenguaje es una acción (expresión, enunciación o palabra escrita) que refiere a un pensamiento como movimiento hacia sí. Todo el lenguaje es acción o recuerdo de acciones (pronunciación, movimiento de la mano o recuerdo de esos sonidos imágenes que uno mismo u otros han realizado), pero esto siempre se realiza con la intención de provocar un pensamiento como un movimiento hacia sí, esta es siempre es su finalidad. Esto es complejo y no se puede explicar extensamente en este espacio.

Existe el lenguaje natural $\mathrm{u}$ orgánico, en donde las acciones poseen algún tipo de vinculación lógico-orgánica con aquello a lo que refieren (como cuando un perro muestra los dientes que -pretendeque se le "entienda" que ha morder si se le acercan, este es lenguaje orgánico porque el perro exhibe los mismos elementos que utilizaría para morder para dar a entender eso al otro, aunque todavía no esté mordiendo o quizá nunca muerda). Este es el lenguaje orgánico en donde existen dos elementos (el mostrar y el sentir/pensar que el otro realiza al ver o percibir lo que se muestra) y también existe el lenguaje creado por los seres humanos que es el que opera por medio de signos arbitrarios ${ }^{10}$. Sin embargo, los ejemplos que antes daba de la persona que mueve los brazos o sube el tono de voz no son en sí casos de "lenguaje no-verbal" o "lenguaje orgánico" en mis propios términos, porque no

${ }^{10}$ El concepto de arbitrariedad introducido por Saussure en el campo de la lingüística en el siglo $\mathrm{XX}$, es uno de los descubrimientos más importantes para comprender al lenguaje. Sobre esto mucho se podría decir... Éste no es un texto dedicado al lenguaje, pero me veo obligado a mencionar algunos aspectos mínimos para poder avanzar. 
necesariamente se hacen con la intención de dar a entender alguna cosa. Solo se hacen, y significan en la medida en que todo lo que hacemos significa. No todo es "lenguaje" si entendemos que el lenguaje es algo que pretende generar alguna otra cosa. El Siglo $\mathrm{XX}$ se ha entusiasmado tanto con el lenguaje que ha hipotasiado todo tipo de ideas bastante curiosas al respecto ${ }^{11}$. En este caso, los movimientos que acompañan a las palabras de la persona que nos acompaña son solo la expresión de las emociones y sentimientos que dichas expresiones verbales, al ser enunciadas, generan en el hablante y que yo puedo percibir porque posiblemente tienen cierta fuerza o magnitud que me permite verlas, que se externen.

Las expresiones verbales del hablante poseen un conjunto de emociones (pensamientos intuitivos) que pueden ser captados por el consultor o filósofo práctico de cierta pericia. Por tanto de lo que se trata aquí es de la utilización de nuestra parte, de acciones corporales de tipo receptivo (percepción) para la captación de las intuiciones del otro, de aspectos emocionales que son movimientos hacia sí, pero que tienen una gran significación en todo lo que está pasando. Este es el primer uso de las acciones corporales que quiero destacar en este texto, que el filósofo perciba al otro y saque provecho de esa percepción. Los contenidos de las percepciones son útiles porque no están presentes en los contenidos discursivos que el otro enuncia, podrían ser claves insustituibles para el desarrollo de un curso de sentido filosófico que de otro modo se podría escapar perdiéndose grandes oportunidades. No basta que el filósofo práctico escuche, también debe oir, ver, oler y sentir, el sentir finalmente es posiblemente una extensión sumamente

\footnotetext{
${ }^{11}$ Yo podría llamar a todo "lenguaje" igual que puedo poner cualquier nombre a lo que "realizamos en general", pero eso no significa que todo lo que hacemos sea igual u opere de la misma manera. Llamar a las expresiones faciales o corporales "lenguaje no verbal" puede ser bastante problemático porque podría dar a entender que tienen significados ocultos o, peor aún, confundirnos completamente respecto a entender las cosas que tenemos delante.
} 
compleja y refinada del -tocarnos- a nosotros mismos como insinúa Antonio Damasio ${ }^{12}$. La percepción del otro es un primer nivel entonces muy importante para el desarrollo de cualquier práctica filosófica, aunque esto posiblemente valga también para todo tipo de disciplinas que impliquen el trabajo con otros como la psicología, educación, sociología, recursos humanos, etc. Este tipo de habilidad debe entrenarse y estudiarse tanto como nuestra capacidad analítca y crítica para la escucha.

Hemos hablado de un primer nivel, el consultor o filósofo práctico percibe. Un segundo nivel es que el consultor o filósofo práctico accione activamente, intervenga en el mundo. El pasaje de lo receptivo a lo activo (de percibir a hacer) normalmente no es tan fácil. Esto es porque lo activo implica a lo receptivo y no al revés, lo activo es un proceso más complejo y elaborado. Por ejemplo, yo no puedo introducir una aguja en el portagujas (que normalmente tiene agujeros para ello), si no veo dónde está el agujero, cómo está posicionado el portagujas, en dónde está la aguja, etc. Puedo ver el porta-agujas y la aguja sin la necesidad de realizar ninguna acción corporal activa, pero no hay forma de que coloque la aguja sin percibir lo que estoy haciendo (además de hacerlo), siempre la acción corporal activa requiere de más procesualidad. $\mathrm{Si}$ anestesiara mi brazo y mi mano (por lo que no sentiría el movimiento que hago) y si además me vendara los ojos, no tendría forma de saber si mi brazo está o no colocando la aguja en el lugar correspondiente. La acción corporal activa requiere de autopercepción de la acción, este es un principo básico de la acción corporal. Por tanto la acción corporal de carácter activa es siempre más compleja y más difícil de realizar tanto para el consultor o filósofo práctico como para el consultante, individuo, grupo u organización con la que se esté trabajando.

12 Algo parecido a cuando dice "el cerebro es la audiencia cautiva del cuerpo" (Damasio, Antonio: Sentir lo que sucede, Editorial Andrés Bello, Chile, 2000, p. 171). 
Pero veamos qué tipo de acciones corporales activas podemos realizar nosotros desde nuestro rol de trabajo. Aquí me estoy refiriendo a todo tipo de gestos corporales, acciones con mi entorno, caras o expresiones. El funcionamiento de esto es interesante porque en este caso estoy realizando ciertas cosas para que sean percibidas. Es posible que yo efectivamente posea los sentimientos que dan o darían pie a tales movimientos de mi rostro, manos, mirada, gestos, etc. Pero yo en estos casos los exacerbo a propósito por el efecto que generan. No puedo ver yo directamente mis caras porque no estoy normalmente frente a un espejo, pero tengo la percepción y la memoria de mis músculos a partir de otras veces que los he movido de esa manera. También observo las reacciones que estos generan en el/los otros. Entonces la percepción de mis caras o gestos es indirecta, pero puedo ir dándoles un curso y esto me permite mantenerlas y darles matices. ¿No podría pensarse que esto una manipulación? Para nada. Todo lo que yo hago en la práctica filosófica es un ping-pong de reacciones e interacciones. ¿Acaso las preguntas de un filósofo lipmaniano, nelsoniano, breniferiano o lahaviano son inocentes? De ninguna manera. Todas ellas se realizan con la intención de generar un efecto. No para que el otro piense como yo pienso o haga lo que yo quiero que haga, pero sí para mover al otro de su "autoculpable minoría de edad" como le llamaba Kant; para sacar al otro de la comodidad del pensamiento cristalizado, cerrado o rutinario. ¿Era Sócrates "auténtico" en todas sus reacciones e interlocuciones? Es difícil decir que Sócrates no sabía acerca del efecto de su exacervado asombro que en ocasiones terminaba en aires sumamente cínicos. Así, Pierre Hadot le atribuye la capacidad de "fingir haberse enamorado", lo que se relaciona con ese acercamiento aparentemente "inocente" sobre las cosas, lo que permitían darse cuenta de todo lo que se ignoraba en relación a la 
vida o a los conceptos que se indagaban ${ }^{13}$. Enfatizar ciertos gestos o exagerar determinadas acciones corporales en la medida en que son percibidas por el otro y no unicamente "porque se sientan", esto refiere también al arte de la práctica filosófica ${ }^{14}$.

Muchas veces lo único que hago es aumentar un poco la sensación que naturalmente una circunstancia dentro de la práctica filosófica me genera y utilizo las mismas "fibras" de lo que a mí mismo me pasa para producir esa reacción. Esto puede pasar, por ejemplo, si yo aumento un poco el asombro que demuestro o la incomodidad que me pueda producir cierta contradicción. En verdad hacer este tipo de cosas requiere de un profundo autoconocimiento porque se trata de utilizar estratégicamente nuestras propias tendencias ${ }^{15}$. Este tipo de reacciones "exageradas voluntariamente" podrían ser de mucha utilidad para las personas con las que estamos trabajando y ayudarlas a ahondar en ciertos puntos de gran importancia gracias a esta reacción performativa. Otras veces, debo ir más allá aún y traer

${ }^{13}$ Así lo explica el autor francés: "El amor irónico de Sócrates consiste pues en fingir haberse enamorado. En la ironía dialéctica Sócrates actuaba de manera similar, planteando sus preguntas, intentando que su interlocutor le comunicara su saber o su sabiduría. Pero de hecho, lo que descubría el interlocutor en ese juego de preguntas y respuestas era su in capaddad para poner algún remedio a la ignorancia de Sócrates..." (Hadot, Pierre: Ejercicios espirituales y filosofía antigua, Siruela, Madrid, 2006, p. 95.).

${ }^{14}$ A esto mismo refiere uno de los lógicos más destacados de México, el filósofo Ariel Campirán, cuando dice que: "El modelaje es una estrategia que consiste en 'hacer ver' al otro a través de variadas acciones inspiradas en el espejo, un desfile de modas, o simplemente en el aparador. El instructor o alguien del grupo modela, cuando actúa (imita, sobreactúa, exagera, repite varias veces, ironiza, etc.) para hacer notar algo" (Campiran, Ariel: Habilidades de pensamiento crítico y creativo, Universidad Veracruzana, Xalapa, s/f, p. 31).

${ }^{15}$ Esto mismo propone por ejemplo mi colega autraliana Narelle Arcidiacono en lo que ella llama "dramatic philosophy", una propuesta sumamente interesante inspirada en el "teatro orgánico" de Grotowsky y en distintas vertientes de la filosofía para niños y la consultoría filosófica. Puede revisarse algo de bibliografía al respecto al final de este texto. 
desde lo profundo de mí alguna reacción que tal vez no me sea tan natural presentar en ese momento. Por ejemplo, algunas veces, al trabajar con adolescentes, y este es un ejemplo que cito a menudo, los jóvenes no desean que se les ponga una "atención tan minuciosa". Si uno se muestra excesivamente interesado por saber de ellos y "entenderlos", esto les genera rechazo y distancia ya que debido a su edad desean principalmente que se los acepte sin analizar demasiado. Sin embargo, la labor filosófica muchas veces requiere de un análisis cuidadoso de lo que se dice o hace, por tanto uno debe asumir una postura de cierto "desinterés", aunque esta no sea nuestra actitud más espontánea. Este pequeño "forzamiento" de uno mismo, permite que el o los otros se abran de una manera espontánea y mucho más natural, mejora los vínculos y finalmente nos permite posiblemente arribar a resultados sumamente diferentes.

En general, este segundo uso de las acciones corporales remite a entenderlas como provocaciones o interpelaciones ${ }^{16}$ hacia el otro. Pero estas interpelaciones no son nada más una simulación o un forzamiento de nosotros mismos. Si así lo hiciéramos, seguramente nuestros interlocutores captarían esta falsedad o autoimposición y tendríamos un resultado más perjudicial que beneficioso. Lo importante es poder hacer uso de nuestros propios recursos para construir una acción de modo que, aunque esta sea generada para producir un efecto, sea a la vez una interpelación auténtica. Y esto es así porque surge del auténtico deseo de que el otro pueda profundizar y conocerse más a sí mismo. En el budismo a esto se lo llama "medios hábiles", en donde se dice que el Buda da forma a su enseñanza según las capacidades del discípulo, la época y la

\footnotetext{
${ }^{16}$ Quien hace uso de este concepto retomado de Levinass para el campo de la filosofía para niños es el profesor José Ezcurdia (Ezcurdia, José: Filosofia para niños, la filosofía frente al espejo, UNAM/Ítaca, México, 2016).
} 
circunstancia ${ }^{17}$. A veces un gran maestro parece estar furioso pero tal vez no lo está de esa manera, a veces parece tranquilo aunque en su interior estallan miles de tormentas. Los llamados "sabios" de las distintas épocas y tradiciones sin duda utilizaban esta forma de la acción corporal conscientemente producida en función de sus efectos, porque sabían de la importancia y gran alcance que tenía. Hoy la práctica filosófica sin duda puede y debe también enriquecerse con este tipo de posibilidades.

El tercer tipo de uso para las acciones corporales dentro de la práctica filosófica que quiero destacar, tiene que ver con solicitar a nuestro consultante, huesped, grupo u organización con la que se esté trabajando, que pueda realizar por sí mismo acciones corporales. Esto incluye también el realizar acciones junto con ellos. Las acciones que el otro podría hacer pueden ser percepciones o intervenciones (acciones corporales de carácter activo); pero en este caso el filósofo práctico ha diseñado o diseña en el curso del trabajo una propuesta para que el otro realice cosas que van más allá del análisis o el discurso, aunque por supuesto pueden combinarse con ellos. En el antiguo México en las escuelas filosóficas de los mexicas (llamadas Cálmecac o Tepochcalli) los tlamatinimes (sabios o filósofos de esta tradición), utilizaban permanentemente este tipo de ejercicios o prácticas:

En esta forma es como los tlamatinime cumplían su misión de '"hacer sabios los rostros ajenos". Y si recordamos, lo dicho acerca de la serie de actos o "costumbres" exteriores guardadas en el Calmécac, veremos que su inflexible rigidez, lo que pudiera llegar a describirse como dureza, iba precisamente dirigida a dar reciedumbre al aspecto dinámico de la personalidad: al corazón. Por medio de esa serie de actos y penitencias disciplinadas, se forjaba el "querer humano", capaz de controlarse a sí mismo. Parece, pues, que lo que buscaban los tlamatinime con su

17 Puede verse Nichiren Daishonin "Enseñanza, capacidad, tiempo y país" disponible on-line en https://www.nichirenlibrary.org/es/wnd1/Content/7 (último acceso 21 de marzo de 2020). 
educación en los Calmécac era perfeccionar la personalidad de sus discípulos en sus dos aspectos fundamentales: dando sabiduría a los rostros y firmeza a los corazones ${ }^{18}$

Así también pasaba en diversas escuelas helenísticas como la escuela estóica o el Jardín de Epicuro. Quienes ingresaban allí se sometían a una ardua disciplina de vida que implicaba el hacer todo tipode cosas y ciertos tipos muy especiales de coordinaciones o coherencias en el curso de sus acciones. Nuestros colegas de aquel entonces, vislumbraban sin lugar a dudas la importancia e irremplazable efecto que genera la realización de acciones corporales para el desenvolvimiento de un proceso filosófico.

Evidentemente, la variedad de acciones corporales que podríamos solicitar o proponer a nuestro visitante o grupo en una sesión de prácticas filosóficas podría ser de la más amplia variedad. Un tipo de trabajo interesante tiene que ver con solicitar percepciones, pedir que el otro mire, toque, huela, escuche o deguste. Esto podría hacerse con objetos específicos que seleccionemos en el momento en función de lo que está sucediendo o que tengamos preparados para la sesión. Desde hace varios años realizo consultorías filosóficas utilizando objetos y resulta bastante interesante por las posibilidades creativas que se dan a partir de la manipulación y el pensamiento analógico. Claro que aquí se trata de una combinación de acciones corporales de carácter activo y receptivo. También es muy interesante crear vinculaciuones perceptivas con diversos contextos que propicien tendencias interesantes en el proceso. Estos contextos pueden estar cerca del espacio en donde estamos trabajando o pueden concertarse previamente con el individuo o grupo para la generación cierto tipo de trabajo. El solo hecho de dialogar en un lugar distinto puede generar una gran diferencia debido a la percepción que opera en forma simultánea a un diálogo

${ }^{18}$ Leon-Portilla, Miguel: Filosofía náhuatl, UNAM, México DF, 1993, p. 2282299. 
o razonamiento. Por supuesto que también podemos solicitar el hacer- de manera activa todo tipo de cosas como parte del trabajo de la sesión. Esto puede hacerse solicitando que el otro haga cosas delante nuestro durante la sesión (sea que hayamos preparado o no tales solicitudes) o a través de -tareas- filosóficas que complementen el trabajo que se realice en los encuentros. Más adelante daré algunos ejemplos de esto. También realizando acciones corporales de manera activa junto con el otro. Esta última posibilidad es sumamente interesante.

Otra clasificación útil para vislumbrar la gran cantidad de posibilidades en todo esto, es la distinción entre acciones corporales críticas y creativas ${ }^{19}$. Realmente hay mucha diferencia entre unas y otras. Intentaré explicarlo con un ejemplo: si solicitamos a una persona con la que estamos trabajando, que se acerque a sus familiares en situaciones cotidianas, pero que en lugar de hacer todo tipo de cosas para complacerlos -como supongamos suele hacer-, solo se mantenga quieta y centrada en su desenvolvimiento individual. Aquí, tenemos un claro ejemplo de una acción corporal crítica que podríamos introducir en un trabajo de consultoria filosofica para tratar el tema ${ }^{20}$, por ejemplo, de la

19 Puede verse Sumiacher, David: "Critical and Creative Philosophical Practices", en Journal of Humanities Therapy, Vol. 7, No. 1, Chuncheon, 2016, pp. 39-70. Aquí se presenta una perspectiva muy amplia de trabajo, no solo considerando las acciones corporales sino todo los diferentes tipos de procesos de la práctica filosófica en sus vertientes críticas o creativas.

${ }^{20}$ Las acciones corporales críticas son sumamente importantes también en el ámbito de la filosofía con organizaciones, pueden ocupar un lugar vital, ya que las organizaciones suelen ser ámbitos en donde operan reglas y objetivos, así como generalmente se vinculan con el mundo del trabajo. No puedo entrar aquí a fondo en las características sistémicas de lo crítico así como lo he venido estudiando, pero el trabajo, las metas, el "deber", el ganarse la vida, etc. todos esos son aspectos que se vinculan a los crítico. También por supuesto se pueden hacer infinidad de cosas creativas en una organización, aquí solo estoy hablando de cierta tendencia. 
complacencia con los demás. Si por el contrario, yo estuviera con un grupo de personas, abordando este mismo tema (la complacencia a los demás), pero quisiera tratarlo por medio de acciones corporales de carácter creativo haría otro tipo cosa. Podría invitarlos a jugar un juego con un objetivo a cumplir -supongamos, resolver un juego de ingenio- pero deberíamos resolverlo de distintas maneras. Primero muy centrados en nosotros mismos, en segundo lugar apoyándonos mutuamente, en tercer lugar apoyándonos exageradamente. Luego de hacer estos juegos analizaríamos y profundizaríamos sobre distintos puntos, pudiendo continuar con otros juegos que continúen trabajando sobre este mismo eje o haciendo otro tipo de cosas, dependiendo esto de la sesión.

En cualquiera de los dos casos (el ejercicio con la familia y el juego con el grupo) yo no estoy "buscando mostrar cómo es que se vive o debe vivir el aspecto de la complacencia con los demás". La práctica filosófica no opera como algunas versiones un poco superficiales del coaching, que pretenden mostrar con la acción corporal de manera literal cierta ruta pre-definida. Para nosotros, las acciones corporales son experimentos, cumplen la misma función que una pregunta solo que operan multidimensionalmente. Cuando se hace una pregunta en la práctica filosófica, nosotros no sabemos la respuesta o no importa tanto -lo que nosotros sabemos-. Escuchamos la respuesta del otro y a partir de allí construimos con ese material. Ahora sucede exactamente igual, no siempre invitamos a la realización de una cierta acción corporal porque ella "muestre lo que se debe hacer". Algunas veces, por el contrario, lo hacemos por su carácter problematizador. A esto lo he llamado problematización con acciones corporales y resulta sumamente interesante en el campo de la filosofía con o para niños o en todo tipo de prácticas filosóficas por su carácter provocador y revelador de significados. 
Lo importante sobre todo, es nuestra habilidad para diseñar estas propuestas, que es una capacidad análoga a la de diseñar buenas preguntas $^{21}$. Las acciones corporales que invitamos a realizar son dispositivos de procesamiento que se engarzan a la procesualidad existente dentro de la o las personas con las que trabajamos. Si el filósofo práctico es alguien preparado para captar ese tipo de magnitudes de proceso, entonces puede imaginar con facilidad qué tipo de acción corporal activa proponer. Podemos, en ocasiones, y con algo de práctica, diseñarlas durante la sesión misma, y luego de unos minutos de escuchar y ver al otro, proponerlas directamente, así como un buen coordinador de talleres filosóficos de cualquier tipo puede improvisar preguntas. Pero también podemos planearlas y por supuesto que las sesiones en la práctica filosófica deben ser planeadas. A veces, debido a cierta hipostación de la idea de la mayéutica, algunas personas piensan que nada puede ser planeado o preparado, pero esto es solamente una visión bastante idealista del asunto. Que exista una planeación no implica por supuesto un direccionamiento total. No es el tema de este texto la planeación, pero en muchas ocasiones existe una especie de "tabú" con el direccionamiento, cuando la buena práctica filosófica, o el buen

${ }^{21}$ Para todo esto es fundamental darnos cuenta con quién estamos trabajando y preguntarnos sobre las personas de manera constante antes y durante el proceso. Eso nos permite reconocer qué tipo de actividad están los demás dispuestos a hacer. Hay personas a las que le encantan los juegos, otras con las que, incluso por el contexto mismo de trabajo, sólo se pueden realizar ejercicios. Algunas veces es interesante trabajar con objetos, otras cambiar radicalmente el contexto. A veces nuestras acciones han de ser sumamente desafiantes y disruptivas otras apenas se salen de lo que normalmente se suele hacer. También es importante captar la visión de las cosas que tienen las personas con las que nos encontramos trabajando para definir el curso de actividades a hacer o proponer. Para el trabajo de la práctica filosófica en el sector salud por ejemplo entender o profundizar sobre cuál es el concepto de fondo al contexto de trabajo es igualmente fundamental para saber qué tipo de disposición tomar y de ejercicios o acciones corporales sugerir. Sobre esto último puede verse Arrigoni, Fabrizio y Nave, Luca: Come in cielo così in terra, Edizioni Unicópoli, Milano, 2013. 
diálogo, es siempre un encuentro de direccionamientos que ya existen y no la ausencia de ellos. De todas formas, siempre puede suceder que, en base a la circunstancia, lo que hayamos planeado deba ser cambiado por completo. Pero eso no significa ese planear haya sido en vano, de allí la famosa frase de Gilles Deleuze que dice que la filosofía al final de cuentas es solo una larga preparación. Planear significa también cuidar, significa pensar en el otro antes de estar ahí, dedicarle tiempo a los demás y dar importancia a lo que hacemos. En muchas ocasiones, sin dedicar excecivas cantidades de tiempo, podemos diseñar muy fructíferas e interesantes propuestas de acciones corporales que terminen generando una significación inusitada.

En todo esto, nuestra captación empática-proyectiva es fundamental, es la misma capacidad que nos permite darnos cuenta si una pregunta estará o no fuera de lugar en un diálogo. También es importante nuestra capacidad para comprender eso que estamos proponiendo, nuestra elocuencia, pasión y la confianza que podamos asentar con aquella o aquellas personas con las que estamos trabajando. Todo eso favorece el lograr que ellos hagan cosas que incluso nunca habían hecho antes. Cuando sean acciones de este tipo, muy recomendable es que el filósofo práctico realice esas acciones en conjunto con las personas, para quitarles el miedo y el pudor que muchas veces genera la acción. Esta es una forma muy interesante de desenvolvimiento del trabajo con acciones corporales dentro de la práctica filosófica ${ }^{22}$. Es como acompañar a

${ }^{22}$ Algunos ejemplos claros en donde esto sucede es cuando se hace una actividad
filosófica en un museo o en un lugar histórico, por ejemplo, pero no solamente
en estos contextos. Las caminatas filosóficas, pueden usarse como medio para
experimentar en conjunto con las personas una percepción renovada respecto de
lo que significan los lugares en donde viven o trabajan cotidianamente. Es
interesante y fructífero trabajar las caminatas filosóficas desde la visión de las
acciones corporales. En un taller filosófico con reclusos o niños de la calle, por
ejemplo, podría ser sumamente importante el realizar tareas manuales,
producción de objetos para vender, etc. junto con ellos, como parte del trabajo de 
alguien a ver el amanecer, como cuando el maestro zen medita junto a sus discípulos o como cuando los que vivían en el Jardín de Epicuro comían en comunidad. Se trata de empatizar con los sentimientos y la "sintonía" de la circunstancia en conjunto con las personas, vivir la experiencia junto a los demás ${ }^{23}$.

Para finalizar, quiero recalcar el hecho de qué así como los pensamientos o palabras desligadas del mundo son vacías y carentes de impacto filosófico; de la misma forma las acciones corporales que no posean un marco de reflexión o diálogo son igualmente huecas y superficiales. Las relaciones que hemos de trazar entre el pensamiento y la acción deben ser nutridas a lo largo de todo el proceso de la práctica filosófica. Para esto podemos intercalar momentos de unas cosas y de las otras. Luego de realizar cualquier tipo de acción corporal, tenemos que preguntar de manera abierta por la experiencia, de modo que las personas puedan expresarnos libremente lo que sienten y piensan y poner en palabras los actos y procesos que anteriormente vivieron. Posteriormente, puedo ir entrando en mayores precisiones y profundizaciones a través del diálogo, todo depende del tipo de sesión y proceso que esté trabajando y con que acometido lo haga $^{24}$. También por supuesto puedo crear ámbitos de reflexión

las sesiones. El -acompañar- a las personas a hacer cosas que tengan un significado profundo que se engarce con los sentidos filosóficos que se vienen construyendo, puede tener una significación sumamente trascendente $\mathrm{y}$ transformar por completo los diálogos y ambientes posteriores.

${ }^{23}$ Otro autor y colega que desde hace tiempo viene trabajando este tipo de enfoque es el español José Barrientos, quien lo aborda desde el concepto de la experiencialidad. Puede verse en la bibliografía algunos textos del autor.

${ }^{24}$ También puede darse el caso de ciertos trabajos con acciones corporales dentro de la práctica filosófica, para los cuales uno tenga que esperar cierto tiempo para dar lugar al momento de conciencia, diálogo, análisis, etc. Podría ser que los procesos que se estén desarrollando, sean sumamente importantes, pero la persona aún no tenga la capacidad de verlo o darse cuenta. Esto debería ser identificado a partir de signos que demuestren dicha dificultad y tratarse 
durante la acción misma, indagando o hablando acerca de puntos interesantes o pertinentes para el proceso.

Generalmente los extremos no son favorables. Para las personas muy proclives a la acción será mejor el pensamiento, para las personas demasiado pensantes hemos de crear valiosas disrupciones en forma de acción corporal dentro del proceso. Ninguno de los dos elementos es tampoco renunciable. Así como no podemos aprender a andar en bicicleta leyedo un libro, ni leer un libro sin tomar el objeto en donde están las letras y palabras y acercarlo para poder verlo; ningún ser humano puede renunciar o realizar cosa alguna, desligándose de los actos y procesos hacia-sí o aquellos de vinculación con la exterioridad. Para poder trabajar con con todo esto, el filósofo práctico debe disponerse a sí mismo por completo, sin escatimar ninguna posibilidad, utilizando todas sus acciones y recursos en pos de profundizar en el quehacer filosófico. Solo este tipo de actitud de total entrega, nos permite abordar un campo de trabajo tan amplio y llegar a niveles de interacción de valor filosófico. ¿Es esto fácil de realizar? Posiblemente no. Seguramente ha de requerir una gran disposición y trabajo consigo mismo de parte del filósofo práctico. Esto en verdad es un valor agregado, pues nos asegura que no estamos cayendo en una labor meramente mecánica o superficial. La práctica filosófica es una apuesta para que la filosofía sea parte de esta sociedad y por ello los filósofos prácticos debemos prepararnos profundamente para poder llevar acabo tan digna tarea. Lo bueno, finalmente, es que las dificultades con que nos encontremos darán testimonio de que lo que podamos obtener realmente vale la pena.

entonces con sumo cuidado. Podrá notarse como la consideración de las acciones corporales dentro de la práctica filosófica abre un abanico enorme de posibilidades y enriquece profundamente nuestra "caja de herramientas". 


\section{Bibliografia}

Arrigoni, Fabrizio y Nave, Luca: Come in cielo così in terra, Edizioni Unicópoli, Milano, 2013.

Austin, John: Cómo hacer cosas con palabras, Ediciones Paidos, España, 1982.

Barrientos, José: "La experiencialidad como respuesta a la tendencia analítica de la filosofía para niños", en childhood \& philosophy, rio de janeiro, v. 12, n. 25, set.-dez. 2016, pp. 519-542. Barrientos, José: Filosofía y espacios sociales, Visión Libros, España, 2013.

Campiran, Ariel: Habilidades de pensamiento crítico y creativo, Universidad Veracruzana, Xalapa, s/f.

Damasio, Antonio: Sentir lo que sucede, Editorial Andrés Bello, Chile, 2000.

Dussel, Enrique: Política de la Liberación. Historia Mundial y

Crítica, Trotta, Madrid, 2007.

Ezcurdia, José: Filosofía para niños, la filosofía frente al espejo, UNAM/Ítaca, México, 2016.

Freire, Paulo: Pedagogía de la indignación. Cartas pedagógicas en un mundo revuelto, Siglo XXI, Buenos Aires, 2012.

Hadot, Pierre: Ejercicios espirituales y filosofía antigua, Siruela, España, 2006.

Harteloh, Peter: "Philosophical walks", en Philosophical Practice. Journal of the APPA. November, New York, 2013, 8.3, pp. 12971302.

Husserl, Edmund: La crisis de las ciencias europeas y la fenomenología trascendental, Crítica, Barcelona, 1991.

Lahav, Ran: Saliendo de la caverna de Platón, Loyev Books, Vermont, 2016.

Leon-Portilla, Miguel: Filosofía náhuatl, UNAM, México, 1993.

Marinoff, Lou: Philosophical Practice, Academic Press, California, 2002. 
Merleau-Ponty, Maurice: Fenomenología de la percepción, Planeta, España, 1994.

Nichiren Daishonin "Enseñanza, capacidad, tiempo y país" disponible on-line en https://www.nichirenlibrary.org/es/wnd1/Content/7 (último acceso 21 de marzo de 2020).

Saussure, Ferdinand: Curso de lingüística general, Planeta, Barcelona, 1984.

Sumiacher, David: "Acts, processes, thought and action in philosophical practice", en Amir, Lydia: New Frontiers of Philosophical Practice: Expanding Boundaries, Cambridge, UK, 2017.

Sumiacher, David: "Critical and Creative Philosophical Practices", en Journal of Humanities Therapy, Vol. 7, No. 1, Chuncheon, 2016.

Sumiacher, David: "La filosofía como una expansión de la vida y los procesos", en Sumiacher, David: Prácticas filosóficas comparadas, Novedades Educativas/Editorial CECAPFI, Buenos Aires, 2020, pp.121-132. 



\section{RESEÑAS BIBLIOGRÁFICAS REVIEWS}


